\title{
Novel sensor design using photonic crystal fibres for monitoring the onset of corrosion in reinforced concrete structures
}

\author{
Colum McCague, Matthias Fabian, Mohammad Karimi, Mikel Bravo, Leszek R. Jaroszewicz, \\ Pawel Mergo, Tong Sun and Kenneth T. V. Grattan
}

\begin{abstract}
In this paper, a novel sensing technique has been designed and investigated for the direct, in-situ detection of steel corrosion distributed in reinforced concrete structures. At present, structural health monitoring (SHM) in reinforced concrete structures is generally focused on monitoring the corrosion risk of the reinforcing steel. It is of significant importance, however, to inform industry of both the onset of corrosion and the corrosion rate as these are key contributors to structural degradation and thus evaluating the service life of the structures. This paper aims to address the above challenges by describing a novel corrosion sensor design using birefringent photonic crystal fibres (PCFs). The technique exploits fully both the birefringence of the fibres for force/pressure measurement and their very low temperature sensitivity to detect the onset of corrosion. This new type of sensor not only determines the onset of corrosion but also allows for better monitoring along the length of a reinforcement bar.
\end{abstract}

Index terms - Optical fibre sensors (OFSs), photonic crystal fibre (PCF), pressure sensor, fibre loop mirror, structural health monitoring

\section{INTRODUCTION}

Over the past century, reinforced concrete has become the most widely used construction material in the built environment [1]. One of the key challenges facing the industry is to design reinforced concrete that will stand the test of time while retaining its integrity during the expected lifespan. One of the main pitfalls of concrete as a construction material is its inability to fully prevent corrosion of the reinforcing steel. While much advancement has been made in terms of protecting against corrosion (i.e. improvements in concrete durability), there is always the risk that corrosion may occur. As a result, corrosion monitoring in concrete structures has now become a major component of the design process. The importance of this was properly recognized in the late 1970s [2], after the degradation of reinforced concrete structures by corrosion was seen as a reality.

C. McCague, M. Fabian, M. Karimi, T. Sun and K.T.V. Grattan are with the Optical Sensors and Instrumentation Group, School of Engineering \& Mathematical Sciences and City Graduate School, City University London, EC1V 0HB, UK. (email: colum.mccague.1@city.ac.uk).

M. Bravo is with the Departamento de Ingeniería Eléctrica y Electrónica Universidad Pública de Navarra, Pamplona 31005, Navarra, Spain. (email: mikel.bravo@unavarra.es)

L. R. Jaroszewicz is with the Military University of Technology, Warsaw, Poland (email: leszek@jaroszewicz.com).

P. Mergo is with the Maria Curie Sklodowska University (UMCS), Lublin, Poland. (email: pawel.mergo@ poczta.umcs.lublin.pl).
At present, a number of techniques are available to assess the likelihood of reinforcement corrosion in concrete structures [35]. These include electrochemical techniques which, under normal working conditions, can give reasonably accurate information relating to parameters such as the steel corrosion rate and the chloride ion concentration. However, with each of these techniques it is difficult to achieve continuous measurement and in most cases rigorous surveys must be carried out manually, on site.

Optical fibre sensors (OFSs) are now becoming increasingly popular in the field of structural health monitoring (SHM). Compared to conventional electrochemical sensing techniques,

OFSs have many characteristics which make them more favourable for SHM including: small size and light weight, immunity to electromagnetic interference, stability of material and good multiplexing capabilities [6]. The concept of SHM using OFSs has been recognized for some time although the industry has been very slow to implement and standardize many such proven forms of measurement. This is mainly due to the high cost of the existing OFS systems and some key issues which require to be addressed to meet industrial standards, such as durability and long term stability.

Over the past decade, the potential for using OFS techniques to monitor the corrosion of metals has been explored. The most popular method has been to replace part of a fibre cladding with the metal under study and monitor changes in transmitted power due to corrosion $[6,7]$. This technique simulates what may happen with nearby steel reinforcement, however, no information of the actual rebar corrosion is provided. In addition, the sensing principle is usually intensity-based, which is known to suffer from limitations of low signal-to-noise ratio and inaccuracy due to fluctuations of the light source. The long period grating (LPG)-based technique has also been reported for monitoring the corrosion of reinforcement in concrete $[8,9]$ which takes full advantage of fibre sensitivity to the refractive index variation of the surrounding environment caused by the changes in ion concentrations [10,11]. More recently, strain measurements using Fibre Bragg Gratings (FBGs) have been reported, in an attempt to directly monitor the corrosion at the steel-concrete interface [12-17].

However, the above OFS techniques can only be used to monitor corrosion at discrete locations where the sensors are installed, but the initiation of corrosion may occur at any point of the rebar, even at locations where there is no sensor installed. Therefore, there is a real need for a monitoring system which is able to alert the onset of corrosion irrespective of its location. This paper aims to address this challenge by using a pressure 
sensing technique based on the polarization characteristics of a polarization-maintaining (PM) photonic crystal fibre (PCFs), in an attempt to accurately determine the onset and development of corrosion along embedded reinforcement bars.

\section{SENSING PRINCIPLE}

Corrosion is an electrochemical process involving chemical reactions and the flow of current between anodic and cathodic sites on the metal surface. When steel corrodes in concrete, rust is deposited at the anodic site (area of steel that has a more positive potential in relation to the cathode) and can occupy a volume many times the volume of the original metal [18]. The expansive forces can eventually exceed the tensile strength of the surrounding concrete, leading to cracking and degradation of the structure. This work is focused on the development of a fully distributed sensor system using a birefringent PCF which is able to capture the expansion force/pressure caused by this rustforming process.

The sensitivity of birefringent PCFs to changes in applied lateral pressure was recently investigated by some of the authors $[19,20]$. Fig. 1 shows the experimental setup, in which an interferometric fibre loop mirror (FLM) is used to convert the birefringence variation of the fibre as a result of the change in lateral pressure into interference pattern shift which is monitored by an optical spectrum analyser (OSA). The peak shifts can be measured and therefore translated into changes in pressure.

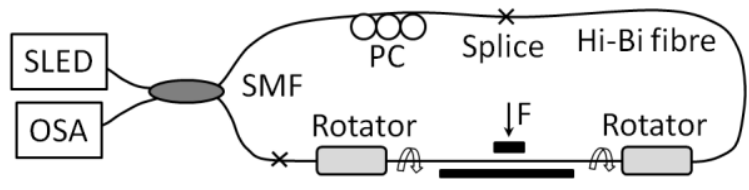

Fig. 1 The setup for characterizing different PCFs based on changes in lateral pressure [19]

The setup consists of a superluminescent light emitting diode (SLED) with emitting wavelength being centered at $1550 \mathrm{~nm}$, a $3 \mathrm{~dB}$ coupler made on singlemode fibre (SMF) which splits the input signal into two waves that counter propagate along completely identical paths and thus experience the same loss. The FLM configuration has shown low insertion loss, polarization independence to input light, broad spectral bandwidth and high resistance to environmental changes as the two waves propagate through identical paths. The extinction ratio of the interferance can be maximized using a polarization controller (PC) within the loop. Rotators allow the adjustment of the fibre orientation until an evenly distributed, maximum sensitivity is achieved. Compared to conventional $\mathrm{HiBi}$ fibres (such as the panda type), HiBi PCFs are less sensitive to changes in temperature making them more suited for environmental applications [21].

The OSA application gives the possibility for measuring the output transmission spectrum $T$ of an optical FLM is described by [22] as:

$$
T=\left[\cos (\varphi / 2) \sin \left(\theta_{1}+\theta_{2}\right)\right]^{2}
$$

where $\theta_{1}$ and $\theta_{2}$ denotes the angle of rotation of the polarization state when propagating light enters the $\mathrm{HiBi} \mathrm{PCF}$ and $\varphi=$ $2 \pi L B / \lambda$ is the phase difference, where $\lambda$ is the guided wavelength, $L$ is the sensor fibre length, $B=n_{\mathrm{x}}(\lambda)-n_{\mathrm{y}}(\lambda)$ is the birefringence, and $n_{\mathrm{x}}(\lambda)$ and $n_{\mathrm{y}}(\lambda)$ are the effective refractive indices of the fast and slow fibre axis, respectively.

The phase difference $\varphi$ in equation (1) changes in response to fibre length and birefringence of which both are affected by mechanical load variation and temperature. Therefore, the wavelength shift $\Delta \lambda$ in the detected transmission spectrum due to temperature and transverse mechanical load variation can be written as:

$$
\Delta \lambda \approx\left(L \frac{\partial B}{\partial T}+B \frac{\partial L}{\partial T}\right) \Delta T+\left(L \frac{\partial B}{\partial F}+B \frac{\partial L}{\partial F}\right) \Delta F
$$

where $\Delta T$ is the temperature variation and $\Delta F$ the mechanical load variation. $\partial L / \partial T$ and $\partial L / \partial F$ describe the elongations of the fibre due to the temperature and mechanical load variation, respectively. These are, however, negligible parameters [23] compared to birefringence change under the force. As PCFs are highly insensitive to temperature variation [21], equation (3) can thus be re-written as:

$$
\Delta \lambda \approx L_{\mathrm{f}} \frac{\partial B_{\mathrm{F}}}{\partial F} \Delta F
$$

where $\mathrm{L}_{\mathrm{f}}$ is the fibre length and $\partial B_{\mathrm{F}} / \partial F$ is the birefringence variation coefficient introduced by the measured lateral mechanical load. $\partial B_{\mathrm{F}} / \partial F$ remains constant depending on the material properties including photoelastic coefficients, Young's modulus and Poisson's coefficient. It is clear from equation (3) that the wavelength shift is directly proportional to the transverse mechanical load.

\section{SENSOR FABRICATION AND INSTRUMENTATION}

For this study, the HiBi PCF type 070107P2 $\left(B=1.446 \times 10^{-3}\right.$ at $1550 \mathrm{~nm}, \varnothing=96 \mu \mathrm{m}, \mathrm{d}=1.4 \mu \mathrm{m}, \mathrm{D}=2.7 \mu \mathrm{m}, \Lambda=3.1 \mu \mathrm{m}$ where $\mathrm{d}$ - small hole $\varnothing, \mathrm{D}$ - large hole $\varnothing$ and $\Lambda$ - distance between adjacent holes) (UMCS, Poland) was chosen due to its higher sensitivity and sharp interference pattern at relatively short lengths. The setup devised by Karimi et al [19] was employed for aligning the sensing fibre before mounting on a steel rebar. A HiBi PCF (250 mm Ø) of $\mathrm{L}=170 \mathrm{~mm}$ was fusion spliced to two lengths of single mode fibres (SMFs) and inserted into the rotators. A schematic of the alignment process is shown in Fig. 2.

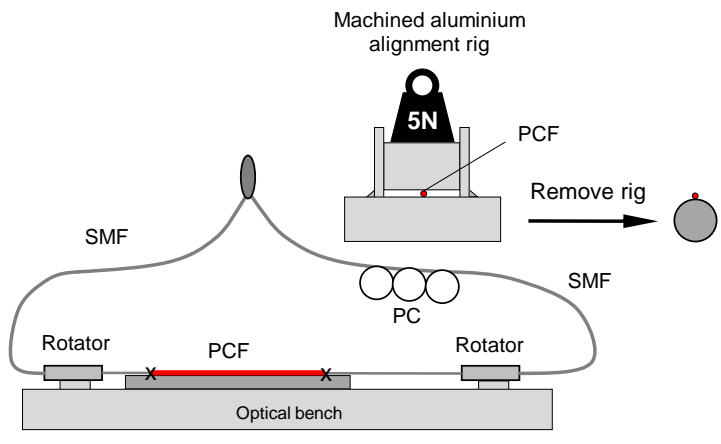

Fig. 2 Schematic of the alignment rig used in this work 


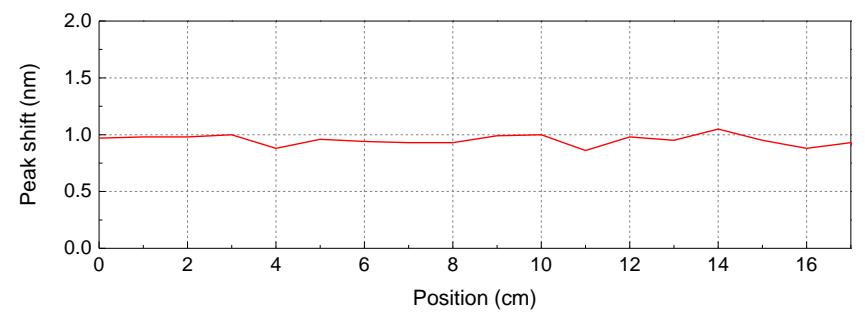

Fig. 3(a) Sensor alignment at a constant load of $5 \mathrm{~N}$ before mounting on the rebar

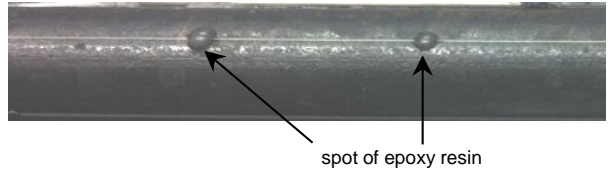

Fig. 4(a) Instrumated rebar with unprotected sensor

It was found that a $5 \mathrm{~N}$ weight applied along $4 \mathrm{~cm}$ of PCF produced a peak shift of $1 \mathrm{~nm}(0.125 \mathrm{~N} / \mathrm{mm} \rightarrow 1 \mathrm{~nm})$. Fig. 3(a) shows the wavelength shift of the sensor when a fixed load of 5 $\mathrm{N}$ is positioned at any point along the length of the sensing fibre. It is noted that the force sensitivity of this distributed sensor is constant irrespective of the location of the force/pressure.

When the alignment was achieved, the rebar was gently positioned underneath the sensing fibre and spot-glued using rapid set epoxy. The sensor response after mounting on the rebar was evaluated by balancing the $5 \mathrm{~N}$ weight at positions between each of the epoxy points. It was found that the sensitivity had reduced slightly near the ends of the sensor as shown in Fig. 3(b). This loss in sensitivity was likely due to an effect induced by the hardened epoxy covering the end splices. Fig. 4 shows the rebar instrumented with a bare (unprotected) sensor and a sensor protected by a thin layer of silicone. The protected sensor did not respond to the $5 \mathrm{~N}$ check due to the reduced sensitivity as a result of the protection, however a positive response occurred by manually exerting additional pressure.

For each sensor, a hairpin loop was made with one of the free SMF ends, directed back to the top of the rebar and fixed into position using epoxy. Isolating heatshrink was used to confine the corroding area of the rebar to the sensing length. All splices were coated with a thin layer of epoxy and located underneath the heatshrink (outside the corroding zones).

\section{ACCELERATED CORROSION TESTS}

Corrosion of steel in concrete in situ in many installations may take months to initiate, even if accelerated in the laboratory by exposing to either $\mathrm{CO}_{2}$ or chloride. As corrosion is an electrochemical reaction, electrical methods can be used to force corrosion [24]. This can be achieved in the laboratory by immersing a concrete-steel specimen in a solution of $\mathrm{NaCl}$ and connecting to a current source as shown in Fig. 5. The experimental program thus developed has been divided into two phases:

\section{Demonstration of the sensing principle (Rebar 1)}

The purpose of this was to transfer the sensing principle demonstrated using laboratory weights, in which a rapid

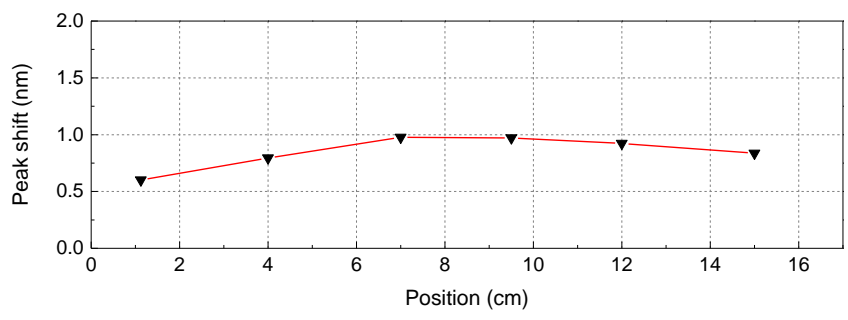

Fig. 3(b) Sensor response to $5 \mathrm{~N}$ after mounting to rebar

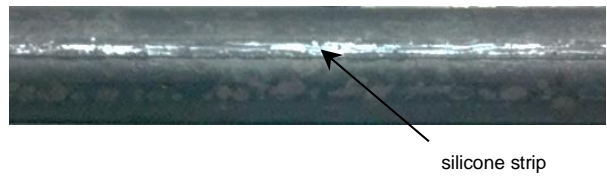

Fig. 4(b) Instrumented rebar with sensor protected by a thin layer of silicone

accelerated corrosion trial of the bare PCF on the instrumented rebar is necessary. For this part, an accelerating current is applied, disconnected, and reapplied again to demonstrate whether peak shifts correspond to changes in corrosion rate.

2. Accelerated corrosion on protected and unprotected PCFs (Rebar 2)

One of the major concerns with any sensing fibre is its durability when exposed to the wet and hardened concrete environment. Thus in this test, by mounting two sensors on opposite sides of the rebar (one bare, and the other protected using the approach illustrated in Fig. 4(a) and 4(b)), a direct comparison could be made between sensors to investigate the effect of using protection (thin silicone coating). The accelerating current would also be monitored throughout the experiment.

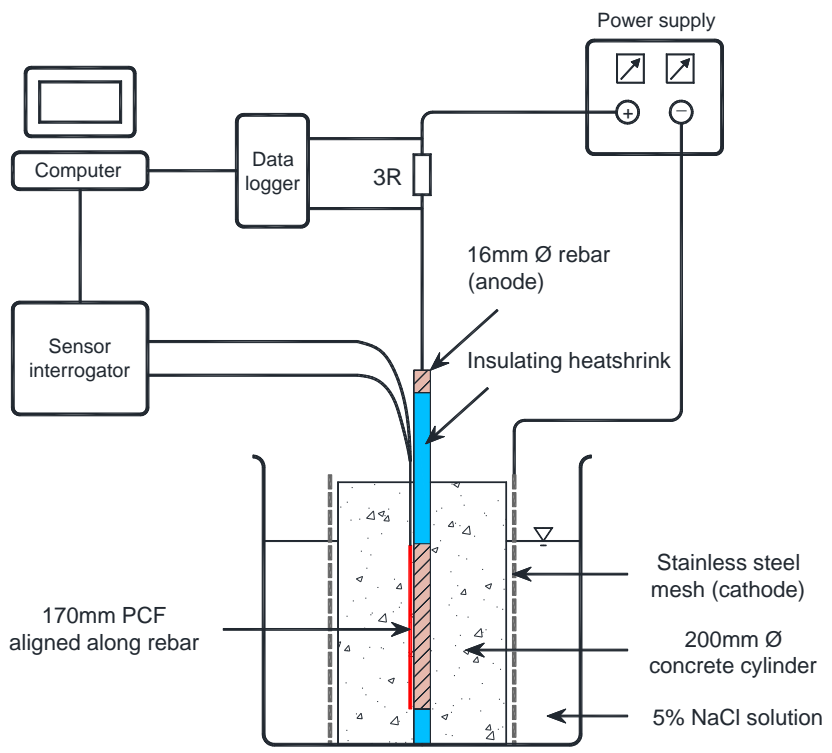

Fig. 5 Accelerated corrosion test setup 
For the concrete, a typical mix was prepared with a cement:sand:gravel weight ratio of 1:2:2.25 and a water/cement ratio of 0.5 (for Rebar 2, salt was added during mixing). The concrete was poured and gently compacted into a prepared cylindrical mould with the sensor/rebar centrally positioned. Both ends of the SMF were connected to a coupler enabling connection to the sensor interrogator (Micron Optics SM125 which is used to replace the OSA and the light source shown in Fig. 1). As the SMF fibres are not polarization maintaining, it was necessary to adjust

the polarization conditions observed to generate the optimum spectral interference pattern (i.e. with maximum extinction ratio so that distinct peaks/valleys can be processed by the peak/valley detection and tracking algorithm). This was achieved by forming small loops with the SMF and arbitrarily adjusting the orientation (similar to the PC in Fig. 2) before fixing in position at the optimum pattern and peak tracking was initiated in the detection system. The concrete was cured at room temperature under moist conditions for two weeks, then demoulded and immersed in water. The peaks were then observed to test the stability under saturated conditions. After the signal had stabilized, salt was added to Rebar 1 and an accelerating voltage of $30 \mathrm{~V}$ was applied between the rebar and the mesh, as shown in Fig 5.

\section{RESULTS AND DISCUSSION}

Rebar 1

Almost immediately after the current was initiated, the peaks of the interference pattern began to shift dramatically and at a steady rate. To confirm that this was due to the corrosion acceleration effect, the current was switched off temporarily at 20 days (as can be seen from Fig. 6).

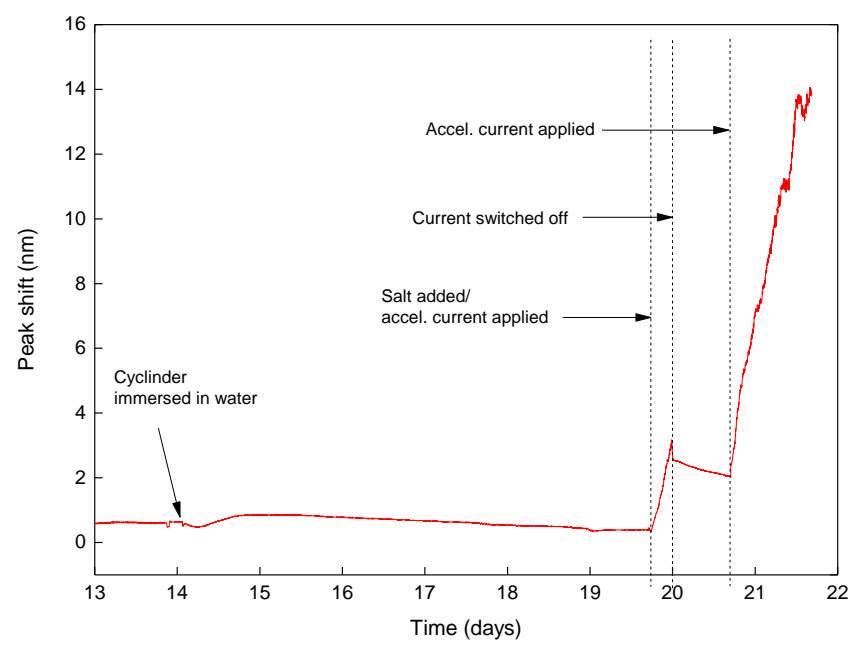

Fig. 6 Validation of sensing concept using the accelerated corrosion test (Rebar 1)

An effect was seen immediately whereby the peaks initially stabilized and then slowly shifted downwards. On the assumption that the detected pressure was from the rapid buildup of hydrated ferric oxide (rust), it would be expected that the pressure would dissipate slightly as the products relax into the concrete pores. After a dormant period of 0.5 days, the accelerating current was reapplied and again positive shifts in the peak positions could be seen almost immediately. The constant pressure induced by the constant accelerating voltage was seen to lead to a linear peak shift response (as expected from Equation 3).

\section{Rebar 2}

As salt was already included within the concrete mix, a significant peak shift was detected by the bare sensor during the curing stage (a gradual peak shift by $6 \mathrm{~nm}$ was seen) as shown in Fig. 7.

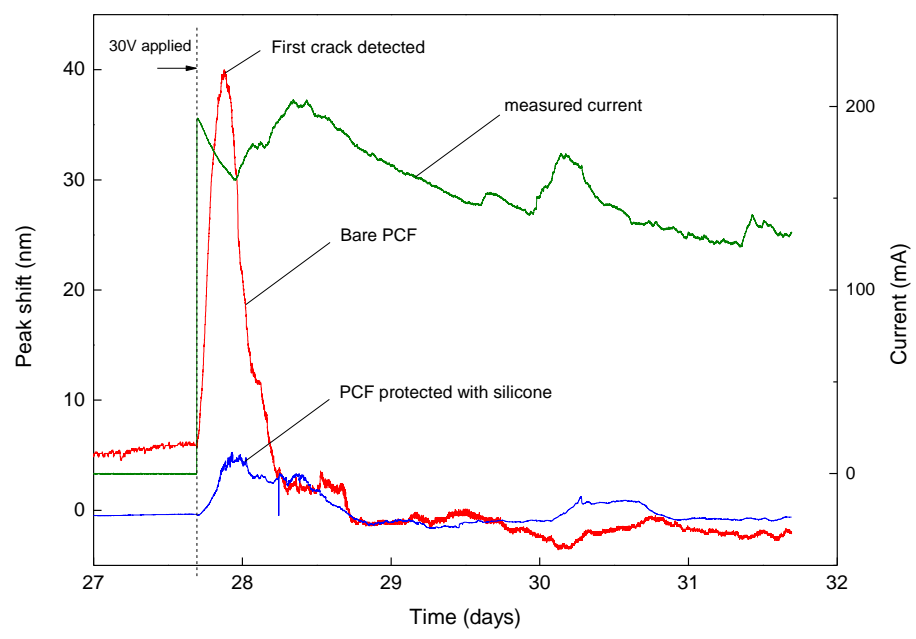

Fig. 7 Behavior of bare and silicone-protected PCFs under accelerated corrosion (Rebar 2)

Once the peaks began to stabilize, the accelerating current was applied. Within minutes, the peak began to shift at a rapid rate. The protected silicone PCF showed an increase in the peak shift at a lower rate compared to that seen for the bare PCF. However, the rate of increase was much greater than in the previous trial (rebar 1) which is likely due to adding salt at the mixing phase. While the sensitivity of the protected fibre is still seen to be lower, there was no delay in the reaction time. In Fig. 7 , the measured accelerating current is also shown. The peak currents correspond to where the crack openings in the concrete are observed, as shown in Fig. 8.

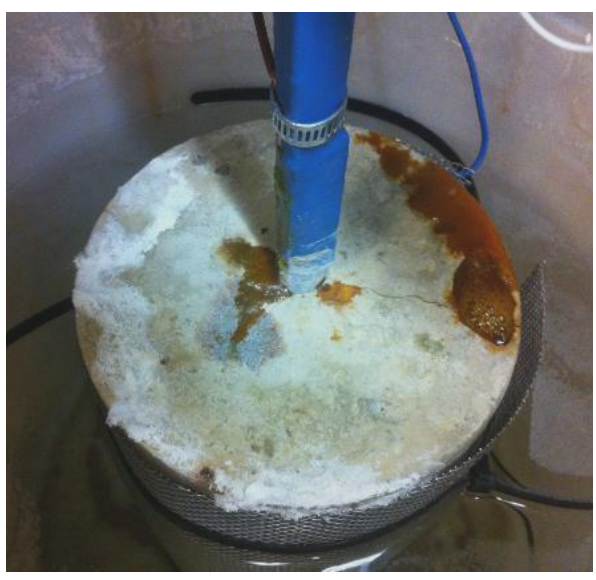

Fig. 8 Photo of corroding cylinder 
The opening of cracks allows more moisture/chloride to penetrate, which in turn leads to an increase in the corrosion rate. After the first main corrosion event, the bare sensor no longer responds to changes in corrosion rate, however, the protected fibre responds to subsequent increases in the corrosion rate (the peak is seen at $\sim 30.5$ days). It was subsequently found that the bare sensor was damaged after the first crack was formed, therefore it could not respond to any further cracking events. However, the protected sensor has been able to survive over the entire testing period and retain sufficient sensitivity to detect subsequent cracking in addition to the first.

\section{CONCLUSIONS AND FUTURE WORK}

This preliminary study has demonstrated that HiBi PCF fibres show excellent potential for effective in situ monitoring of the corrosion of reinforcing steel in concrete from the point where such corrosion has been initiated. Very useful information can thus be obtained from the sensors, showing a direct relationship between the measured pressure and the corrosion rate of the rebar. This direct sensing approach not only provides information on the corrosion rate itself but also acts as an ideal sensor for crack detection. The data obtained also allows an analysis of the direct stresses induced by the expansive corrosion reaction, which enable a furtherance of the better understanding of many aspects of topical civil engineering research. With a constant sensitivity achieved over the whole length of the sensing fibre, there is significant potential to embed such sensors along an entire length of rebar at selected points to determine stresses and cracking that occurs.

Future work will evaluate these effects in greater detail, allowing correlations to be made to weight loss and the measured corrosion current. Other aspects of sensor development are ongoing with a particular focus on sensor protection, corrosion location identification and determination of corrosion arte, thus creating sensor design improvements to meet industrial needs.

\section{AKNOWLEGEMENT}

The authors would like to thank the support from the EU COST Action TD1001: Novel and Reliable Optical Fibre Sensor Systems for Future Security and Safety Applications (OFSeSa) for this joint research undertaken. The support of the George Daniels Educational Trust is gratefully acknowledged. The authors are grateful to UMCS, Poland for supplying the PCF used in this study.

\section{REFERENCES}

[1] E. M. Gartner, "Potential improvements in cement sustainability," in 31st Cement and Concrete Science Conference, Imperial College London, 2011, p. 13.

[2] L. Bertolini, B. Elsener, P. Pedeferri et al., "Preface," Corrosion of Steel in Concrete: Prevention, Diagnosis, Repair, pp. v-vii: Wiley, 2004.

[3] Ø. Vennesland, M. Raupach, and C. Andrade, "Recommendation of Rilem TC 154-EMC: "Electrochemical techniques for measuring corrosion in concrete"-measurements with embedded probes," Materials and Structures, vol. 40, no. 8, pp. 745-758, 2007.
[4] C. Andrade, and C. Alonso, "Corrosion rate monitoring in the laboratory and on-site," Construction and Building Materials, vol. 10, no. 5, pp. 315-328, 1996.

[5] H. W. Song, and V. Saraswathy, "Corrosion monitoring of reinforced concrete structures - A review," International Journal of Electrochemical Science, vol. 2, no. 1, pp. 1-28, Jan, 2007.

[6] S. Dong, Y. Liao, and Q. Tian, "Intensity-based optical fiber sensor for monitoring corrosion of aluminum alloys," Applied Optics, vol. 44, no. 27, pp. 5773-5777, 2005.

[7] M. Benounis, and N. Jaffrezic-Renault, "Elaboration of an optical fibre corrosion sensor for aircraft applications," Sensors and Actuators B: Chemical, vol. 100, no. 1-2, pp. 1-8, 2004.

[8] Y. Wang, and H. Huang, "Optical fiber corrosion sensor based on laser light reflection," Smart Materials and Structures, vol. 20, no. 8, 085003, 2011.

[9] W. Hu, H. Cai, M. Yang et al., "Fe-C-coated fibre Bragg grating sensor for steel corrosion monitoring," Corrosion Science, vol. 53, no. 5, pp. 1933-1938, 2011.

[10] K. R. Cooper, J. Elster, M. Jones et al., "Optical fiber-based corrosion sensor systems for health monitoring of aging aircraft," in IEEE Systems Readiness Technology Conference, Valley Forge, USA, 2001, pp. 847-856.

[11] S. K. A. Kaed Bey, C. C. C. Lam, T. Sun et al., "Long period grating pair chloride ion sensor for early corrosion prevention," Journal of Physics: Conference Series, vol. 85, no. 1, 012022, 2007.

[12] X. Zhao, Y. Cui, H. Wei et al., "Research on corrosion detection for steel reinforced concrete structures using the fiber optical white light interferometer sensing technique," Smart Materials and Structures, vol. 22, no. 6, 065014, 2013.

[13] M. R. Abu Hassan, M. H. Abu Bakar, K. Dambul et al., "Opticalbased sensors for monitoring corrosion of reinforcement rebar via an etched cladding Bragg grating," Sensors (Basel), vol. 12, no. 11, pp. 15820-6, 2012.

[14] X. Zhao, P. Gong, G. Qiao et al., "Brillouin Corrosion Expansion Sensors for Steel Reinforced Concrete Structures Using a Fiber Optic Coil Winding Method," Sensors, vol. 11, no. 11, pp. 1079810819, 2011.

[15] Z. Zheng, X. Sun, and Y. Lei, "Monitoring corrosion of reinforcement in concrete structures via fiber Bragg grating sensors," Frontiers of Mechanical Engineering in China, vol. 4, no. 3, pp. 316-319, 2009.

[16] S. K. T. Grattan, P. A. M. Basheer, S. E. Taylor et al., "Corrosion induced strain monitoring through fibre optic sensors," Journal of Physics: Conference Series, vol. 85, no. 1, 012017, 2007.

[17] S. K. T. Grattan, S. E. Taylor, S. Tong et al., "Monitoring of corrosion in structural reinforcing bars: performance comparison using fiber-optic and electric wire strain gauge systems," Sensors Journal, IEEE, vol. 9, no. 11, pp. 1494-1502, 2009.

[18] J. P. Broomfield, "Corrosion of Steel in Concrete," Corrosion of Steel in Concrete: Understanding, Investigation and Repair, pp. 79: CRC Press, 2006.

[19] M. Karimi, M. Fabian, T. Sun et al., "Transverse force sensitivity of photonic crystal fibres," in Sensors, 2012 IEEE, 2012, pp. 4.

[20] M. Karimi, T. Sun, and K. Grattan, "Design evaluation of a high birefringence single mode optical fibre-based sensor for lateral pressure monitoring applications," Sensors Journal, IEEE, vol. PP, no. 99, p. 8, 2013.

[21] Z. Chun-liu, Y. Xiufeng, L. Chao et al., "Temperature-insensitive Interferometer using a highly birefringent photonic Crystal fiber loop mirror," Photonics Technology Letters, IEEE, vol. 16, no. 11, pp. 2535-2537, 2004.

Y. Liu, B. Liu, X. Feng et al., "High-birefringence fiber loop mirrors and their applications as sensors," Applied Optics, vol. 44, no. 12 , pp. 2382-2390, 2005.

[23] B. Culshaw, "The optical fibre Sagnac interferometer: an overview of its principles and applications," Measurement Science and Technology, vol. 17, no. 1, pp. 1-16, 2006.

[24] T. El Maaddawy, and K. Soudki, "Effectiveness of impressed current technique to simulate corrosion of steel reinforcement in concrete," Journal of Materials in Civil Engineering, vol. 15, no. 1, pp. 41-47, 2003. 